The ASTROPHYSICAL JOURNAL, 371:L41-L44, 1991 April 10

(C) 1991. The American Astronomical Society. All rights reserved. Printed in U.S.A.

\title{
DYNAMIC MAGNETIC RECONNECTION AT AN X-TYPE NEUTRAL POINT
}

\author{
I. J. D. CRAIG \\ University of Waikato, Department of Mathematics, Private Bag, Hamilton, New Zealand \\ AND \\ A. N. MCCLymont \\ University of Hawaii, Institute for Astronomy, 2680 Woodlawn Drive, Honolulu, HI 96822 \\ Received 1990 December 4; accepted 1991 January 18
}

\begin{abstract}
The relaxation of a two-dimensional "X-type" neutral point magnetic field disturbed from equilibrium is considered. Perturbations are shown to possess well-defined azimuthal modes which allow an exact determination of the magnetic annihilation rate. Free magnetic energy is dissipated by oscillatory reconnection which couples resistive diffusion at the neutral point to global advection of the outer field. The decay of azimuthally symmetric $(m=0)$ modes - the only modes associated with topological reconnection-is limited by the dissipation time scale of the "fundamental" $(n=0)$ mode with no radial nodes. This mode decays over typically 100 Alfvén times. An analytic treatment shows that the oscillation and decay time scales couple according to $\tau_{\text {osc }} \approx 2 \ln S$ and $\tau_{\text {decay }} \approx \tau_{\text {osc }}^{2} /\left(2 \pi^{2}\right)$, where $S$ is the Lundquist number $\left(4 \pi / c^{2}\right) v_{\mathrm{A}} R / \eta$ and the times are in units of $R / v_{\mathrm{A}}$, with $R$ the distance from the neutral point to the boundary, $v_{\mathrm{A}}$ the Alfven speed at the boundary, and $\eta$ the resistivity.
\end{abstract}

Subject headings: hydromagnetics — stars: flare — stars: magnetic — Sun: activity — Sun: corona Sun: flares

\section{INTRODUCTION}

The exotic properties of X-type neutral points have played a central role in the development of solar flare theory as well as in theories of coronal heating (e.g., Parker 1979). A key issue is the rate of magnetic field annihilation in the vicinity of the neutral point: can energy be released fast enough to account, for example, for the explosive phase of the solar flare? Early studies of the merging of antiparallel magnetic fields showed that pure resistive diffusion is much too slow (Sweet 1958; Parker 1963). However, Petschek (1964), in his celebrated paper, argued that magnetic energy can be released at a significant fraction of the Alfvén speed provided account is taken of the "advection region" surrounding the neutral point. The semiquantitative Petschek solution has attracted much critical debate over the years (e.g., Vasylunias 1975), but the physical viability of the underlying mechanism seems secure.

To date, most discussions of magnetic merging have followed the steady state approach adopted by early workers (see Forbes \& Priest 1987). Apart from the lack of dynamical completeness, a central difficulty with this approach is matching the large-scale advection of the global field to the highly localized-and approximately treated-diffusion region surrounding the neutral point. The purpose of this Letter is to present a formally exact analytic solution for the timedependent relaxation of a perturbed magnetic field toward an $\mathrm{X}$-type potential field. For small departures from equilibrium, the problem can be reduced to that of determining the radial dependence of a scalar flux function. The construction of a set of eigenfunctions of the linearized magnetohydrodynamic equations then allows an exact determination of the magnetic annihilation rate. We concentrate here on the purely radial $m=0$ modes which correspond to topological reconnection. The "fundamental" mode with no radial nodes $(n=0)$ decays most slowly: to high accuracy, its decay time is given by $\tau_{\text {decay }} \approx\left(2 / \pi^{2}\right)(\ln S)^{2}$ in units of the Alfvén time scale of the advection region, where $S$ is the Lundquist number determined by the magnetic field strength at the boundary. The decay time is that associated with fast reconnection-typically about 100 Alfvén times in the solar corona.

The general problem is formulated in $\S 2$, and solutions are presented in $\S 3$. Section 4 provides a summary of our findings. A full exposition of the analysis summarized in this Letter will be communicated in subsequent publications (e.g., Craig \& Watson 1991).

\section{ANALYSIS}

\subsection{Background and Assumptions}

We consider the relaxation of a magnetic field perturbed from an X-point potential field. Our analysis is performed in plane cylindrical coordinates, the field lines being coplanar (no $B_{z}$ component) and anchored to a circular radial boundary. We neglect gas pressure, ignore viscosity, and assume constant resistivity in a uniform density background plasma.

Figure 1 shows a typical solution of the linearized X-point problem. The sequence of images shows field lines undergoing oscillatory reconnection, with each row constituting an oscillation cycle. After three cycles, the field has relaxed from its initial stressed state to very close to a potential field.

\subsection{Formulation of the Equations}

The equilibrium potential field $\boldsymbol{B}_{\boldsymbol{E}}$ can be written in cylindrical polars as

$$
\boldsymbol{B}_{E}=r(\sin 2 \phi \hat{\boldsymbol{r}}+\cos 2 \phi \hat{\boldsymbol{\phi}}),
$$

where we have scaled the radius and field strength in units of the coronal length $R$ and the magnetic field strength $B_{0}$ at the boundary, $r=1$, at which we take the field lines to be held fixed. We interpret $R$ as the size of a typical coronal magnetic structure. 




FIG. 1.-Evolution of field lines in the "fundamental" $(m=0, n=0)$ mode for $\eta=10^{-3}$, obtained by numerical solution of the eigenequation (2.6). The number above each plot, $\omega t / 2 \pi$, gives the phase of the oscillation; three complete cycles are shown, during which the amplitude of the perturbation decreases by a factor of around 60 . Note that during the oscillation the field lines reconnect as they pass through the origin, so resistivity is essential to this mode. The initial vertical "current sheet" begins reconnecting, and the inertia of the flowing plasma carries the system past the equilibrium configuration, till a (weaker) horizontal "current sheet" is formed at phase $=\frac{1}{2}$. A much weaker vertical "current sheet" returns at the end of the first cycle. After three cycles, the system is very close to its equilibrium neutral point configuration.

The problem is much simplified by working with the vector potential, or flux function, $\psi(r, \phi)$, where $\boldsymbol{B}=\boldsymbol{\nabla} \times(\psi \hat{z})$. The equilibrium flux function has the form $\psi_{E}=-\frac{1}{2} r^{2} \cos 2 \phi$. The evolution of $\psi$ is governed by the induction equation

$$
\frac{D \psi}{D t}=\eta \nabla^{2} \psi
$$

where $D / D t \equiv \partial / \partial t+v \cdot \nabla$ is the Lagrangian time derivative, we have scaled time in units of $R / v_{\mathrm{A}}, v_{\mathrm{A}}=B_{0} /(4 \pi \rho)^{1 / 2}$ is the Alfvén speed at the boundary, and the dimensionless resistivity $\eta$ is simply the inverse Lundquist number (loosely, the inverse magnetic Reynolds number) at the boundary.

In the case of zero diffusion, the magnetic potential is advected with the flow and the field topology cannot change. However, finite resistivity allows field line reconnections that induce fundamental changes in the field topology. The diffusion term is typically very small $\left[\eta \approx O\left(10^{-14}\right)\right.$ for solar coronal conditions], but it always dominates in the region of the neutral point. Accordingly, the diffusion region, no matter how localized, is central to the evolution of the global topology.

The dynamics of the problem are determined by the momentum equation, which we write in dimensionless form as

$$
\frac{D v}{D t}=-\nabla^{2} \psi \nabla \psi
$$

The right-hand side is the Lorentz force $j \times \boldsymbol{B}, \boldsymbol{j}=-\nabla^{2} \psi \hat{z}$ being the dimensionless current density.

\subsection{The Linearized System}

We linearize equations (2.2) and (2.3) and combine them into a single differential equation for $\psi$. The result is

$$
\ddot{\psi}-\eta \nabla^{2} \psi=\left|\nabla \psi_{E}\right|^{2} \nabla^{2} \psi,
$$

where $\psi$ now denotes a first-order perturbation on the flux func- tion $\psi_{E}$, and we have used dots for time derivatives. To analyze equation (2.4) we assume separation of variables and write

$$
\psi(r, \phi, t)=e^{\lambda t} f(r) e^{\mathrm{im} \phi} .
$$

The eigenequation for $f(r)$ is then

$$
r\left(r f^{\prime}\right)^{\prime}=\left[\lambda^{2} /\left(1+\eta \lambda / r^{2}\right)+m^{2}\right] f,
$$

where prime means $\partial / \partial r$ and we have used the fact that $\left|\nabla \psi_{E}\right| \equiv\left|B_{E}\right| \equiv r$. The decomposition in $\phi$ is always validthe trigonometric functions are complete-but the $r$ - $t$ separation can be justified only a posteriori from the properties of the eigenmodes.

On the outer boundary $r=1$ we set the perturbation $\psi=$ constant which "freezes in" the field, since $\delta B_{r} \equiv$ $(1 / r) \partial \psi / \partial \phi \equiv 0$. Furthermore, equation (2.4) then shows that $\nabla^{2} \psi$ vanishes on the boundary, so the $\boldsymbol{j} \times \boldsymbol{B}$ force and therefore also the velocity vanishes. Thus we study a closed system, with no magnetic flux or plasma entering or leaving the volume. Although an arbitrary constant (indeed any curl-free function) can be added to $\psi$ in equation (2.4), $f(1)=0$ is the only allowable boundary condition in equation (2.6).

\section{THE SOLUTION}

\subsection{Introduction}

We first discuss the properties of equation (2.4), then point out the special character of the $m=0$ mode, which we concentrate on for the remainder of this Letter. Next we mention the general solution for $m=0$ and examine its properties for several specific cases. Finally, we show how analytic solutions for the $m=0$ mode can be obtained in the asymptotic limit $\eta \rightarrow 0$ and point out that these solutions are in fact valid for all $\eta$ of practical interest.

\subsection{Properties of the Equation}

Equation (2.4), the most general statement of the linearized problem, reveals both a diffusive and a wavelike character. First, consider the region close to the origin, that is $r \ll r_{c}$, where $r_{c}$ is the usual skin depth

$$
r_{c} \equiv a \eta^{1 / 2},
$$

and $a \approx O(1)$ does not depend strongly on $\eta$. Then equation (2.4) reduces to the diffusion equation, $\psi=\eta \nabla^{2} \psi$, whose properties are well known and understood: in particular the equation possesses azimuthal modes $\psi_{m}$ with a Bessel function radial dependence, $\psi_{m}=J_{m}\left[(-\lambda / \eta)^{1 / 2} r\right] e^{i m \phi}$. Diffusion is ultimately responsible for dissipating the magnetic and kinetic energy in our system.

In the advection region $\left(r \gg r_{c}\right)$, equation (2.4) reduces to the wave equation, $\ddot{\psi}=r^{2} \nabla^{2} \psi$. The rate of propagation of information is governed by the wave speed $v_{\mathrm{A}} \propto\left|B_{E}\right| \propto r$ which makes the signal travel time logarithmic in $r$. Thus a disturbance on the outer boundary propagates into the diffusion region $r<r_{c}$ in a time $\delta t \approx\left|\ln r_{c}\right| \approx \frac{1}{2}|\ln \eta|$. This fundamental dependence determines the time scale for the oscillatory relaxation we find to be a universal feature of the solution.

\subsection{Reconnection and the $m=0$ Modes}

It is important to understand that $m=0$ perturbations are the only modes which allow topological reconnection. Field line reconnection is synonymous with dissipation of current at the neutral point. Close to the neutral point $\left(r \ll r_{c}\right)$, the 
radial dependence of the current $j_{z}=-\nabla^{2} \psi$ is given by $J_{m}\left[(-\lambda / \eta)^{1 / 2} r\right]$. The only Bessel function which is nonzero at the origin is $J_{0}(x)$ so the $m=0$ modes alone can reconnect field lines. The higher $m$ modes are nontopological in that they dissipate current in the outer field but not at the origin. More generally, in contradistinction to the $m=0$ modes, higher $m$ disturbances can decay by nonresistive means, such as damping of fluid motions by finite viscosity.

In view of their special significance, we henceforth consider only the $m=0$ modes. On physical grounds we expect the $m=0$ decay rate to be limited by the disturbance of longest radial wavelength, i.e., the "fundamental" $(n=0)$ mode with zero nodes in the radial direction.

\subsection{The General Solution for $m=0$}

Although equation (2.6) must be integrated numerically in general, for $m=0$ it reduces to a hypergeometric equation (Hassam 1990). This formally confirms the existence and uniqueness of the $m=0$ solution and provides an independent check on the computed reconnective modes.

Figure 2 shows the first few radial eigenfunctions and eigenvalues for $m=0$ and $\eta=10^{-3}$, a relatively large resistivity chosen to render certain features of the solution more apparent. The wavelike character of the solution in the advection region is evident; these waves propagate into the diffusion region where they are absorbed. As expected, the interface between the diffusion and advection regions (the point at which $\psi$ begins dropping from its central plateau) occurs at $r_{c} \approx \eta^{1 / 2}=10^{-1.5}$.

For the $m=0$ mode, there is no perturbation on the radial component of the field. The perturbation on the azimuthal field is given by $\delta B_{\phi}=-\partial \psi / \partial r$. The effect of superposing this perturbation on the equilibrium field is illustrated in Figure 1, discussed earlier.

\subsection{Time Scales and Their Dependence on $S$}

A key issue is the reconnection rate and its dependence on resistivity. Figure 3 shows the oscillation period and decay

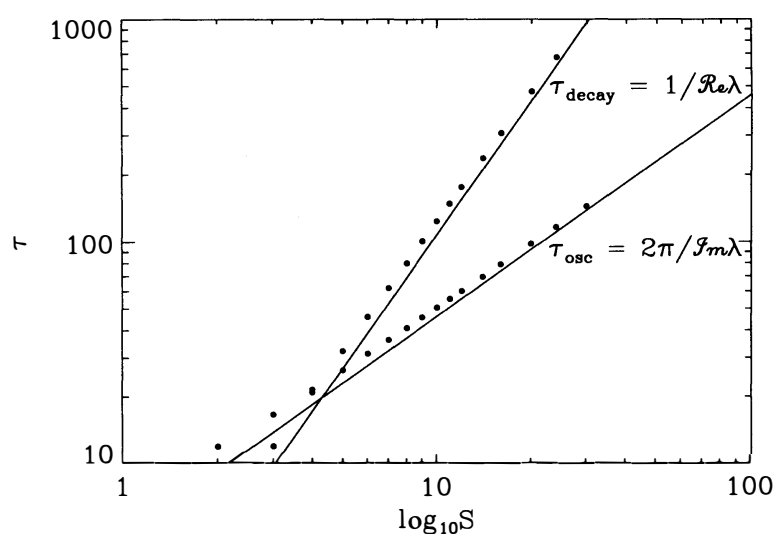

FIG. 3.-Plot of the decay time $1 / \operatorname{Re}(\lambda)$ and oscillation period $2 \pi / \operatorname{Im}(\lambda)$ against $\log _{10} S$ (on a $\log -\log$ plot) where $S=1 / \eta$ is the Lundquist number. The points indicate results from numerical solutions of eq. (2.6), while the lines represent the analytic results (3.6) and (3.8). For Lundquist numbers of less than $10^{4}\left(\eta>10^{-4}\right)$, the perturbation decays in less than one oscillation. For $S \approx 10^{14}$ (a plausible coronal value), the oscillation period is around 60 Alfvén times, and the perturbation decays in about three oscillations.

time for the slowest decaying $m=0$ mode as a function of the Lundquist number $S$. Points indicate numerical solutions, while the straight lines denote the analytic solutions derived below. The oscillation period grows nearly linearly with $\log S$, while the decay time is approximately quadratic in $\log S$. For $S \lesssim 10^{4}$, the decay time is shorter than the oscillation period, so the oscillation is heavily damped.

\subsection{Analytic Solutions}

We now show that the form of the eigenfunctions and the essential time scales can be derived by simple analytic arguments which both reinforce and describe the numerical results obtained above. To do this we assume that the diffusion region can be represented as a boundary layer of thickness $r_{c}$, as defined in equation (3.1) with the scaling parameter $a$ of order unity.
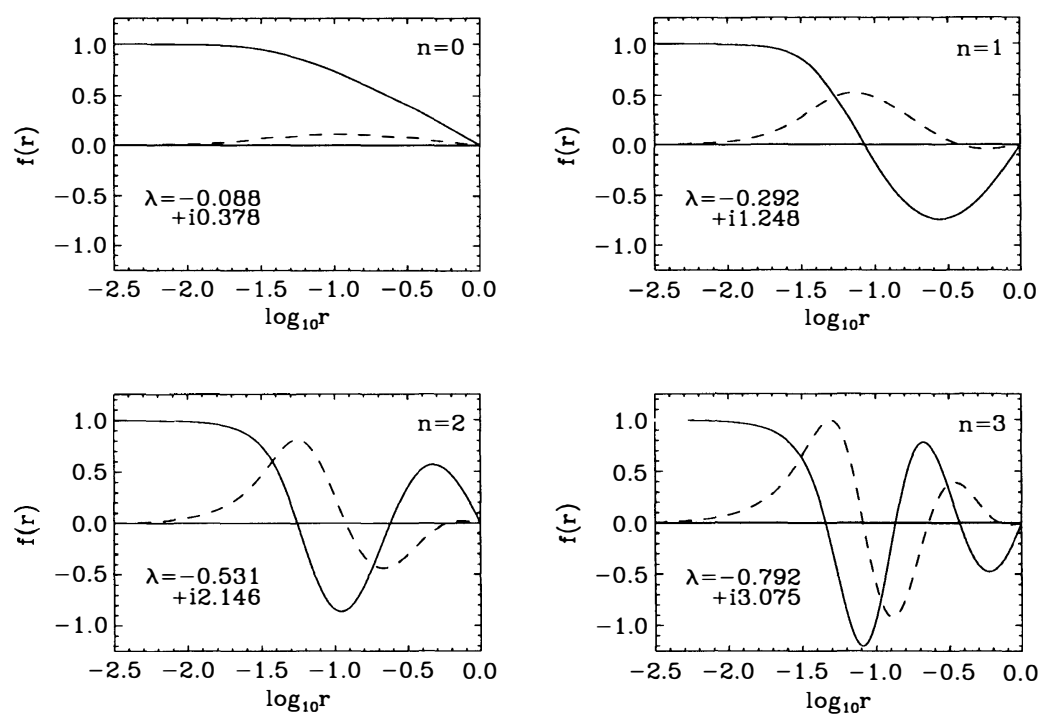

FiG. 2.-Real (solid line) and imaginary (dashed line) parts of the eigenfunctions for $\eta=10^{-3}, m=0$, and $n=0,1,2,3$, where $m$ and $n$ are the number of azimuthal and radial nodes respectively. 
Written explicitly in terms of real and imaginary parts, with

$$
\lambda=-\alpha+i \omega ; f(r)=f_{R}(r)+i f_{I}(r),
$$

equation (2.4) becomes

$$
\begin{array}{r}
{\left[\begin{array}{cc}
{\left[\left(\omega^{2}-\alpha^{2}\right)+\left(r^{2}-\eta \alpha\right) \nabla^{2}\right]} & -\omega\left[2 \alpha+\eta \nabla^{2}\right] \\
+\omega\left[2 \alpha+\eta \nabla^{2}\right] & {\left[\left(\omega^{2}-\alpha^{2}\right)+\left(r^{2}-\eta \alpha\right) \nabla^{2}\right]}
\end{array}\right]\left[\begin{array}{l}
f_{R} \\
f_{I}
\end{array}\right]} \\
=\left[\begin{array}{l}
0 \\
0
\end{array}\right] .
\end{array}
$$

For $m=0$, appropriate boundary conditions are $f_{R}(0)=1$ and $f_{I}(0)=f_{R}(1)=f_{I}(1)=0$.

Now, if $\eta$ is reduced, the diffusion region contracts and the wave domain extends inward toward the origin. For small enough $\eta$, the problem becomes advection-dominated, and we are led to seek standing wave solutions with imaginary eigenvalues but real eigenfunctions. Thus, as a zeroth order approximation we attempt a solution with $\alpha=f_{I}=0$. Then in the wave domain

$$
\left(\omega^{2}+r^{2} \nabla^{2}\right) f_{R}=0 \Rightarrow f_{R}=\sin (\omega \ln r) \quad r \gtrsim r_{c} .
$$

This solution may be matched at $r_{c}$ to the simplest boundary layer solution that satisfies the inner boundary condition, i.e., $f_{R}=1$, to yield

$$
\frac{1}{2} \omega \ln \eta \approx-\left(n+\frac{1}{2}\right) \pi,
$$

where $n$ is the number of radial nodes and we have assumed $\eta \ll a$. Therefore the oscillation frequency of the fundamental mode, $n=0$, is given by $\omega \approx \pi /|\ln \eta|$, or

$$
\tau_{\mathrm{osc}} \approx 2 \ln S \text {. }
$$

To determine the damping rate clearly requires an inner approximation that maintains more structure, and finite current, in the boundary layer. Retaining small but finite $\alpha$ and $f_{I}$ in the above equations leaves the outer solution unchanged but gives

$$
\left(\alpha+\eta \nabla^{2}\right) f_{R} \approx 0 \Rightarrow f_{R}=J_{0}\left[(\alpha / \eta)^{1 / 2} r\right] \quad r \lesssim r_{c}
$$

as a better boundary layer approximation. This solution allows us to match the currents in the inner and outer regions at $r=r_{c}$, with the result $\alpha=(\omega / a)^{2}$. The fact that this relationship agrees well with the numerical results of Figure 3 confirms that the matching radius $r_{c}$ scales as expected. Apparently, the numerical results are well described by the retrospective choice $a=2^{1 / 2}$, or $r_{c}=(2 \eta)^{1 / 2}$. This gives

$$
\tau_{\text {decay }} \approx \frac{2}{\pi^{2}}(\ln S)^{2},
$$

in excellent agreement with the numerical results as $\eta \rightarrow 0$.

\section{CONCLUSIONS}

We have shown that the relaxation of a perturbed X-type neutral point magnetic field can be reduced to an eigenvalue problem in which the advection and diffusion regions are treated exactly. It is also possible to construct approximate analytic solutions by matching inner and outer solutions at the interface between the diffusion and advection regions. These solutions are asymptotically exact for small $\eta$ but remain quite accurate for $\eta$ as large as $O\left(10^{-3}\right)$, and so suffice for the decription of virtually every plasma of astrophysical interest.

We have emphasized that only the $m=0$ azimuthal modes correspond to topological reconnection; all other modes are current-free in the vicinity of the neutral point. The reconnection process is oscillatory: inertial overshoot of the plasma carries more flux through the neutral point than is required for static equilibrium.

The reconnection rate scales as $(\ln \eta)^{2}$, so the reconnection is "fast," as opposed to the "slow" reconnection which depends on a power of $\eta$. Thus "fast" relaxation need not be continuously "driven" as in steady state models but occurs naturally as a result of diffusive-advective coupling even for small disturbances. The free magnetic energy is dissipated in typically 100 Alfven times, based on the Alfvén speed at the boundary, during which time the plasma undergoes several oscillations through the neutral point. By virtue of our closed outer boundary which reflects waves, all the free energy is converted into heat.

For typical solar parameters, the dissipation time scale is of order several minutes to an hour. This is sufficiently rapid to account for thermal energy release in the gradual phase of a flare but could be too slow to explain the impulsive phase time scale. However, the time scale is short enough to ensure that long-lived current sheets cannot exist in the corona; therefore, the corona should be substantially force-free.

We thank Jim Drake, Yuhong Fan, George Fisher, Adil Hassam, and Paul Watson for insightful discussions. A. N. M. is supported by NSF grant ATM88-22366 and NASA grant NAGW-864.

\section{REFERENCES}

Craig, I. J. D., \& Watson, P. G. 1991, in preparation

Forbes, T. G., \& Priest, E. R. 1987, Rev. Geophys., 25, 1583

Hassam, A. B. 1990, BAAS, 22,853

Parker, E. N. 1963, ApJ, 138, 552

1979, Cosmical Magnetic Fields (Oxford: Oxford Univ. Press)

Petschek, H. E. 1964, in AAS-NASA Symposium on the Physics of Solar

Flares (NASA SP-50), ed. W. N. Hess, p. 425
Sweet, P. A. 1958, in IAU Symposium 6, Electromagnetic Phenomena in Cosmical Physics, ed. B. Lehnert (Cambridge: Cambridge Univ. Press), p. 123

Vasylunias, V. M. 1975, Rev. Geophys. Space Phys., 13(1), 303 